\title{
State of the art and new perspectives for non- invasive point-of-care testing
}

\begin{abstract}
Point-of-care (POC) analytical technology has empowered patients with a more convenient, rapid, reliable, and cost-effective diagnostic tool for self-management of health. In order to realize the effective disease management and appropriate therapeutic interventions in real time, there is a great need for a POC health monitoring system which allows non-invasive, accurate, convenient, easy-to-use, painless, and very fast measurements. By reviewing some current technologies of POC, we believe for the next generation of POC testing, home testing devices with non-invasive testing approach, accurate results, and real-time measurements will be the new trends.
\end{abstract}

Keywords: cancer, chronic disease, point-of-care, non-invasive, healthcare, home testing
Volume I Issue I - 2016

\author{
Yunqing Du,' Ming L Wang ${ }^{2}$ \\ 'Interdisciplinary Engineering Program, Northeastern University, \\ USA \\ ${ }^{2}$ Civil and Environmental Engineering, Northeastern University,
} USA

Correspondence: Yunqing Du, Interdisciplinary Engineering Program, College of Engineering, 400SN, Northeastern University, 360 Huntington Ave, Boston, MA, USA. 021 I5, Tel 617-373-3010, Email du.yu@husky.neu.edu

Received: September 07, 2016 | Published: September 30, 2016
Abbreviations: POC, point-of-care; POCT, point-of-care testing; FDA, US food and drug administration; NBM, non-invasive hemoglobin measurement; $\mathrm{CE}$, conformité européene; $\mathrm{CDN}$, certified dialysis nurse; CAP, college of american pathologists; CLIA, clinical laboratory improvement amendments

\section{Introduction}

Point-of-care (POC) testing enables rapid diagnosis or monitoring of chronic conditions that can facilitate clinical decisions. Such technologies have potential to significantly change the healthcare and diagnostic pathways. Patients can obtain immediate results in hospital, physician's office, or even at home. This approach saves patients' tremendous amount of time, resulting in a faster application of efficient treatment or referral for further investigation. Nowadays, POC devices are well established in clinical practice for early detection and management of disease. ${ }^{1}$ Several well-known applications include the in-home blood glucose monitoring system for diabetes management, ${ }^{2}$ the prothrombin time test for anti-coagulation level measurement, ${ }^{3,4}$ and the pregnancy test, ${ }^{5}$ to name a few. However, most of the approaches are invasive process which involves the use of blood. Patients are usually unwilling to conduct blood test due to annoying pains and massive scarring formation that is developed from finger pricks or blood draw. These disadvantages hinder POC technologies from more extensive application in prospect. Thus, the aim of this review is to reveal the future trends in POC technologies, which is the real-time and non-invasive disease diagnosis and monitoring system for home use. Furthermore, the cost-effective point-of-care testing (POCT) devices should possess comparable accuracy and sensitivity to conventional hospital laboratory analyzers, so reliable results can empower patients to track their health progress (Figure 1).

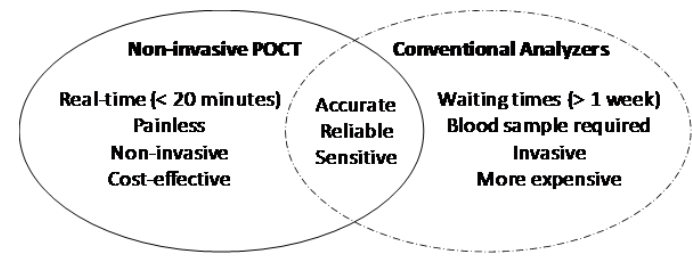

Figure I Comparison between non-invasive point-of-care testing (POCT) and conventional analyzers.

\section{Discussion}

POC testing has already been utilized for screening patients from cancers and other chronic diseases. According to cancer facts and figures 2016, nearly 600 thousand Americans are expected to die of cancer in 2016, which translates to about 1,630 people per day. ${ }^{6}$ It is essential to diagnose cancers at their early stages before it had the chance to get too big or spread, so the improvement in survival reflects both the earlier diagnosis of certain cancers and improvements in treatments. It would have saved millions people's lives if an early warning system of cancers are available. In addition, the prevalence of chronic diseases is drawing billions cost from the government budget for health care each year, ${ }^{7}$ and such huge burden can be mitigated if the non-invasive POC testing system can help monitor disease-related biomarkers for disease monitoring and control. The real-time results will dramatically decease the time for diagnosis and institutional treatment, reduce in-patient hospital stay, eliminate laboratory test analysis, and improve cost-effectiveness. Such successful health care system will provide better health to more people at lower cost on a continuous basis.

Unfortunately, whole blood, plasma, or serum is still the most common sample taken to measure different substances due to limitations of current technologies. The results can help detect health problems in their early stages, when treatments or lifestyle changes may work best. Typically, the current practice of implementing health care is to apply routine checkups with physical and blood tests. Through this approach, doctors can check for certain conditions, or diagnose diseases. As to now, blood tests are well recognized for diabetes diagnosis, also for screening and early warning of skin cancer relapse, ${ }^{8}$ prostate cancer, ${ }^{9}$ and breast cancer. ${ }^{10}$ The procedure is usually quick and easy, however, sometimes it may also cause some short-term discomfort, even vasovagal reactions for some people such as fainting or dizziness due to the blood drawn. Moreover, the routine blood test is usually scheduled yearly or even less frequently. For people newly diagnosed with aggressive cancer, if the routine checkup time happens to exceed their best therapy times, then the treatment options are very limited and they are suffering more harms. As a result, in order to realize the effective disease management and appropriate therapeutic interventions in real time, there is a great need for a POC health 
monitoring system which allows non-invasive, accurate, convenient, painless, and more importantly, very fast measurements.

Many companies and research institutes have devoted great

Table I Comparative aspects of point-of-care testing (POCT) devices attention to the development of non-invasive or minimally invasive disorders monitoring systems. A couple technologies are introduced and compared as examples to discover future perspectives of POC applications (Table 1).

\begin{tabular}{|c|c|c|c|c|}
\hline Product & Characterizations & Company & Technology & Device certification \\
\hline $\begin{array}{l}\text { Dexcom continuous glucose } \\
\text { Monitoring system }\end{array}$ & $\begin{array}{l}\text { Minimally invasive } \\
\text { Real-time }\end{array}$ & Dexcom \& Google & Enzyme-base electrochemical sensor & No \\
\hline Gluco-watch & $\begin{array}{l}\text { Non-invasive } \\
\text { Real-time }\end{array}$ & $\begin{array}{l}\text { Bio control technology } \\
\text { inc. }\end{array}$ & $\begin{array}{l}\text { Near infrared transmission } \\
\text { spectroscopy }\end{array}$ & No \\
\hline Nbs 200 & $\begin{array}{l}\text { Non-invasive } \\
\text { Real-time }\end{array}$ & Or sense & Occlusion technology & Ce, Cdn, Fda $50 \mathrm{I}(\mathrm{K})$ \\
\hline Color genomics kit & $\begin{array}{l}\text { Non-invasive requires } \\
\text { Labaratory tests }\end{array}$ & Color genomics inc. & Sequencing technology & CAP \& CLIA \\
\hline $\begin{array}{l}\text { Oraquick advance rapid } \\
\text { HIV-I/2 Antibody }\end{array}$ & Non-invasive real-time & $\begin{array}{l}\text { Orasure technologies } \\
\text { inc. }\end{array}$ & Enzyme immunoassays & FDA CLIA-Waived \\
\hline
\end{tabular}

\section{Minimally invasive devices}

A mini continuous blood glucose monitor developed jointly by DexCom and Google can be attached to the skin in contact with subcutaneous tissue and measure sugar levels in interstitial liquid, ${ }^{11}$ With a price of $\$ 150$, the selling point of the device is the accessibility of real-time glucose data for diabetes management. However, it is not approved by the U.S. food and drug administration (FDA) to stand alone as a reliable blood glucose monitoring method. ${ }^{12-14}$ Compared to fingertip blood tests, the interstitial liquid test is minimal invasive and causes less discomfort to patients. Nevertheless, it is still not an ideal approach due to its potential side effects, such as skin irritation and infections. Thus, latest generations are focusing on transferring this technology into more user-friendly disposable devices, such as bandage and contact lens. ${ }^{15}$

\section{Optical detector}

Targeting on the non-invasive glucose detection, Bio control Technology Inc. attempted to use infrared light by illuminating the light on patients' arm, and then collecting the light which scattered back out through the skin for measurement. Nevertheless, this tissue spectroscopy method has low signal-to-noise ratio, and signal is easily interfered by physiological changes of each individual ${ }^{16}$ Without a FDA approval, accuracy is always the critical factor for POC use. Another non-invasive blood analytes monitoring device, the NBM 200 system, was developed by or Sense. Routine operation can be performed by combining the optical measurement platform with a finger attached ring-shape sensor probe. By temporarily occluding blood flow in the finger, a newly created blood dynamics can generate informative transmission signal across the finger. The product has received $\mathrm{CE}$ and $\mathrm{CDN}$ approval and received FDA 510(k) clearance in 2013 for non-invasive hemoglobin testing. This is a huge step forward as the non-invasive POC tests are finally deliverable for home use.

\section{Saliva-based sensing system}

Recently, a non-invasive method using saliva as an indicative fluid is being explored to manage chronic diseases. ${ }^{17,18}$ Using saliva for disease diagnostics and health surveillance is a promising approach since saliva has been found to contain constituents that reflect the diseased or physiological state of the human body. ${ }^{19-21}$ A genetic screening test, called color genomics, is offering an at-home DNA saliva test to detect gene mutations linked to breast and ovarian cancer. Instead of one single biomarker analysis, the comprehensive analysis of 30 genes endow this technology with a more rigorous determination. Unfortunately, the expensive $\$ 250$ test kit is still a step away from the real POC. Although consumers can collect their saliva sample easily and non-invasively at home, they need to mail the sample out for analysis. Here, the real-time diagnostic result or test report is still missing.

On the other hand, a more successful and relatively mature saliva-based test kit was launched by OraSure Technologies, Inc. in early $21^{\text {st }}$ for the in-home HIV test. A quick home testing using Ora Quick Advance Rapid HIV-1/2 Antibody can reveal results within 20 minutes. Furthermore, the oral fluid detection was approved by FDA in 2002, and an average sensitivity of $95 \%$ and specificity of $99.8 \%$ verified from the screening tests. ${ }^{22}$ Despite its inspiring performance, a long list of limitations of the test also indicates the difficulties to control accuracy in real practice. ${ }^{23}$ However, it gives us an idea about how critical the two factors: accuracy and real-time performance can affect the prosperity of similar technologies for POC testing.

\section{Conclusion}

Overall, new trends are formed in the POC setting that hammer at high accuracy, sensitivity, convenience, reliability, non-invasion, and most importantly, the real-time measurements of disease-related biomarkers. The non-invasive monitoring technology offers patients a simple and fast way to measure the real-time disease-related biomarker levels frequently as needed with no pain, stress-free, no risk of infection, and non-scarring. For the future perspectives of POC applications, accurate home testing, non-invasive testing, and realtime performance would be the key factors to evaluate the feasibility of the developed technology. To fulfill the objectives, future research and publications need to focus on studying or guiding the standard accuracy and capabilities of non-invasive POC technologies to the home setting. These contributions would certainly help push technology forward and improve health outcomes through selfmanagement.

\section{Acknowledgements}

None. 


\section{Conflict of interest}

The author declares no conflict of interest.

\section{References}

1. Lash TB. Point-of-care diagnostic testing; 2016.

2. Olansky L, Kennedy L. Finger-stick glucose monitoring issues of accuracy and specificity. Diabetes Care. 2010;33(4):948-949.

3. Heu M, Welborn T, Nagykaldi Z. In adult patients on warfarin, does home self-testing of prothrombin time and/or international normalized ratio provide the same outcomes compared to testing by a home health nurse or in a clinical setting? Journal of Oklahoma State Medical Association. 2016;109(3):99-100.

4. Heneghan C, Ward A, Perera R, et al. Self-monitoring of oral anticoagulation: systematic review and meta-analysis of individual patient data. Lancet. 2012;379(9813):322-334.

5. Childerhose and Macdonald. Health consumption as work: the home pregnancy test as a domesticated health tool. Soc Sci Med. 2013;86:1-8.

6. Society, Cancer facts \& figures 2016; 2016.

7. Estimates of diabetes and its burden in the united states, in National diabetes statistics report 2014, Centers for disease control and prevention, Atlanta, GA, USA: CDC; 2012.

8. UK Blood test may give early warning of skin cancer relapse; 2016.

9. Society prostate cancer prevention and early detection; 2016

10. Blood test could give early warning of breast cancer; 2014.

11. Effectiveness and safety study of the dexcom ${ }^{\mathrm{TM}} \mathrm{G} 4$ continuous glucose monitoring system. Clinical Trials gov. 2012.
12. FDA. Dexcom G4 PLATINUM (Pediatric) continuous glucose monitoring system - P120005/S002; 2016.

13. FDA. Modifications to devices subject to premarket approval (PMA) The PMA supplement decision; 2015.

14. Mark R Burge, Stephen Mitchell, Alison Sawyer, et al. Continuous glucose monitoring: the future of diabetes management. Diabetes Spectrum. 2008;21(2):112-119.

15. Google and dexcom team up to dramatically improve CGM; 2015.

16. Hopkins tissue spectroscopy for glucose measurement; 2006.

17. Du Y, Zhang W, Wang M. Sensing of salivary glucose using nanostructured biosensors. Biosensors (Basel). 2016;6(1):10.

18. Du Y, Zhang W, Wang M. An on-chip disposable salivary glucose sensor for diabetes control. J Diabetes Sci Technol. 2016;10(6):1344-1352..

19. Wong. Salivary diagnostics for oral cancer. $J$ Calif Dent Assoc. 2006;34(4):303-308.

20. Wong. Salivary diagnostics powered by nanotechnologies, proteomics and genomics. J Am Dent Assoc. 2006;137(3):313-321.

21. Castagnola M, Picciotti PM, Messana I, et al. Potential applications of human saliva as diagnostic fluid. Acta Otorhinolaryngol Ital. 2011;31(6):347-357.

22. Lyons J. FDA-approved rapid HIV antibody screening tests 2008 . Hearth research \& educational trust, USA: Springer; 2008.

23. Summary of safety and effectiveness data OraSure Technologies, Inc; 2010 . 